\title{
How do physicians and nurses differ in their perceived barriers to effective enteral nutrition in the intensive care unit?
}

\author{
Masoumeh Mirhosiny ${ }^{1}$, Mansour Arab ${ }^{2}$, Parvin Mangolian Shahrbabaki ${ }^{3,4}$ \\ ${ }^{1}$ Critical Care Nursing, Payambar Azam Hospital, Kerman; ${ }^{2}$ Critical Care Nursing, Faculty of Nursing and Midwifery, Bam University of Medical Sciences, Bam; \\ ${ }^{3}$ Nursing Research Center and ${ }^{4}$ Razi Faculty of Nursing and Midwifery, Department of Critical Care Nursing, Kerman University of Medical Sciences, Kerman, Iran
}

Background: Patients hospitalized in intensive care units are susceptible to chronic malnutrition from changes in protein and energy metabolism in response to trauma. Therefore, nutritional support, especially enteral nutrition, is one of the most important treatment measures for these patients. However, there are several barriers in the hospitals in treating patients with enteral nutrition. This study was performed to compare the perceptions of care providers (physicians and nurses) on the barriers to enteral nutrition in intensive care units.

Methods: This was a cross-sectional descriptive and analytic study. This study included 263 nurses and 104 physicians in the intensive care units of Kerman University of Medical sciences, in south east of Iran. A questionnaire of enteral nutrition barriers in intensive care units was used. IBM SPSS ver. 19 was used to analyze data.

Results: There was a significant difference between the two groups in the three subscales of intensive care units $(P=0.034)$, dietician support $(P<0.001)$ and critical care provider attitudes and behavior $(\mathrm{P}=0.031)$. There was also a significant difference between having completed educational courses and the score of enteral nutrition barriers in the two groups $(P<0.05)$; the people who received an educational course had a better perception of enteral nutrition barriers.

Conclusions: Physicians and nurses agreed with the perception of enteral nutrition barriers, but there was a difference in their perception on some barriers. Strategies such as in-service training and increasing the knowledge and skills of physicians and nurses can reduce these differences.

Key Words: critical care; enteral nutrition; intensive care; intensive care units; nurse; physician

\section{INTRODUCTION}

Nutrition plays an important role in patients with a chronic and acute condition, but some of these patients cannot feed themselves because of metabolic stress and/or being in an unconscious state. Without proper nutritional support, these patients are at serious risk for malnutrition [1-3]. Impaired immune function, increased risk of sepsis, and weakening of respiratory muscles are some of the outcomes of malnutrition that can result in extended use of a mechanical ventilation machine [4]. Enteral nutrition involves administering nutrients by nasogastric, oral-gastric, or percutaneous tubes into the duodenum or jejunum and is a suitable strategy for patients hospitalized in intensive care units [5-9]. Enteral nutrition should

\section{Original Article}

Received: February 15, 2021

Revised: June 23, 2021

Accepted: June 24, 2021

\section{Corresponding author}

Parvin Mangolian Shahrbabaki

Faculty of Razi School of Nursing

and Midwifery, Kerman University of

Medical Sciences, Haft-Bagh

Highway, Kerman, Iran

Tel: +98-34-3132-5192

Fax: +98-76-1691-3555

E-mail:mangolian167@yahoo.com

Copyright (C) 2021 The Korean Society of Critical Care Medicine

This is an Open Access article distributed under the terms of Creative Attributions Non-Commercial License (https:// creativecommons.org/li-censes/by-nc/4.0/) which permits unrestricted noncommercial use, distribution, and reproduction in any medium, provided the original work is properly cited. 
be the first and foremost nutrition therapy in patients whose gastrointestinal tract is functioning well. Despite the importance of enteral nutrition in intensive care units, this method is associated with some obstacles [8].

Several types of factors prevent the implementation of recommended guidelines in clinical practice, such as those related to individuals, social issues, and organizations [10]. Understanding these barriers helps to identify the gap between recommended guidelines and practices and enables the development of strategies to overcome these barriers $[11,12]$. The researchers reported that patients receive on average less than $60 \%$ of their prescribed calories and protein [13].

A literature review on enteral nutrition revealed the gap between the recommendations of evidence-based guidelines and what is achieved in intensive care units [11,12,14,15]. A qualitative analysis, using interviews with nutritionists, physicians, and nurses, identified several barriers to adequate nutrition in the intensive care units, such as guidelines, implementation process, institutional factors, individual provider behavior, and patient clinical conditions [16]. In another study, the most important barriers included an insufficient supply of feeding pumps, insufficient enteral formula in the unit, and difficulties in obtaining small bowel access in patients that do not tolerate enteral nutrition [11]. Views and attitudes of medical and nursing practitioners in Australia on the barriers to nutrition intervention in intensive care units showed that there are competing priorities when caring for patients, and the implementation of nutrition therapy is influenced by the practitioner roles and expectations [15]. A study in England reported that only half of the patients under intensive care received the necessary nutrients. Some reasons for this included a delay in the start and prescription of enteral nutrition, disconnection of nutrition due to surgical and diagnostic procedures, gastrointestinal intolerance, and no standard protocol of enteral nutrition for nurses [14].

Barriers can vary based on the different sites, local context, and available facilitators in hospitals [17]. This indicates that the effect of each barrier differs according to profession, supporting the need to better understand the barriers faced by each profession and how the barriers differ according to the context $[7,10]$. In addition, some barriers may be common across intensive care units, but the frequency and magnitude of these barriers may differ due to the unique elements of the local context and available facilitators. Therefore, critical care providers have to be aware of enteral nutrition barriers [13]. This study aimed to compare the views of nurses and physi-

\section{KEY MESSAGES}

- By identifying the barriers to enteral nutrition in patients admitted to intensive care units, it is possible to plan, implement and prevent complications in these patients.

- Awareness of the treatment team's perspective can increase optimal nutritional care of the intensive care patients.

- Reducing the difference between the perceptions of physicians and nurses plays an important role in removing enteral nutrition barriers and providing appropriate enteral nutrition in intensive units.

cians on enteral nutrition barriers in the intensive care units of hospitals Kerman University of Medical sciences.

\section{MATERIALS AND METHODS}

\section{Ethical Statement}

This study was approved by the Ethics and Research Committee of Kerman University of Medical Sciences (No. IR.Kmu. REC. 1396, 1665), After obtaining the necessary permits, the researcher referred to the study setting at different shift works and coordinated with participants for the study. After specifying eligible participants, sufficient information about study aims, their importance, and confidentiality was provided for participants. The researcher tried not to refer during rush hours and visiting hours in the department to prevent any interference in patients' treatment process and also to ensure that all participants were mentally ready and had enough time to answer the questionnaire. The questionnaires were given to the participants and then delivered after completion.

\section{Study Design and Setting}

This descriptive-analytical cross-sectional study was conducted on intensive care units of teaching hospitals affiliated to Kerman University of Medical Sciences University of Medical Sciences. These centers are among the largest ones in in south east of Iran. This study lasted from May to July 2020.

\section{Sample Size and Sampling}

The study population consisted of 110 physicians and 270 nurses working in intensive care units in 2020. The inclusion criteria for nurses included a B.S or higher degree, while the inclusion criteria for physicians included a specialty or higher degree. Overall, 104 physicians and 263 nurses participated in 
the study, and the response rate was $94.5 \%$ for physicians and $97.4 \%$ for nurses.

\section{Instrument}

A demographic information form and questionnaire on the barriers to delivery of enteral nutrition were used. The form collected demographic and background information including age, sex, marital status, work experience, and work experience in an intensive care unit.

The barriers to the delivery of enteral nutrition questionnaire developed by Cahill et al. [18] in the U.S. consisted of 26 items and five dimensions. The five dimensions are as follows. (1) "Guideline recommendations and implementation strategies" includes: I am not familiar with our current guidelines for nutrition in the intensive care unit, current scientific evidence supporting some nutrition interventions is inadequate to inform practice, the language of the recommendations of the current guidelines for nutrition are not easy to understand, the current guidelines for nutrition are not readily accessible when I want to refer to them, no feeding protocol in place to guide the initiation and progression of enteral nutrition, and current feeding protocol is outdated. (2) "Intensive care unit resources" includes: not enough nursing staff to deliver adequate nutrition, enteral formula not available on the unit, and no or not enough feeding pumps on the unit. (3) "Dietician support" includes: waiting for the dietitian to assess the patient, dietitian not routinely present on weekday patient rounds, no or not enough dietitian coverage during evenings, weekends and holidays, and not enough time dedicated to education and training on how to optimally feed patients. (4) "Delivery of enteral nutrition" includes: delay in physicians ordering the initiation of enteral nutrition, waiting for physician/ radiology to read $\mathrm{x}$-ray and confirm tube placement, frequent displacement of feeding tube, requiring reinsertion, delays in initiating motility agents in patients not tolerating enteral nutrition (i.e. high gastric residual volumes), delays and difficulties in obtaining small bowel access in patients not tolerating enteral nutrition (i.e. high gastric residual volumes), in resuscitated, hemodynamically stable patients, other aspects of patient care still take priority over, and nutrition therapy not routinely discussed on patient care rounds. (5) "Critical care provider attitudes and behavior" includes: non-intensive care unit physicians (i.e. surgeons, gastroenterologists) requesting patients not be fed enterally, nurses failing to progress feeds as per the feeding protocol, feeds being held due to diarrhea, fear of adverse events due to aggressively feeding patients, feeding being held too far in advance of procedures or operating room visits, and general belief among intensive care unit team that provision of adequate nutrition does not impact on patient outcome. The 5-point Likert scale was used and included the following: not at all important (score=1), somewhat unimportant (score=2), neither important nor unimportant (score $=3$ ), somewhat important (score $=4$ ), and very important (score $=5$ ). The minimum and maximum scores were 26 and 130, respectively. The content validity index was determined (0.92) and the Cronbach's alpha coefficient was calculated to be 0.81 .

\section{Data Analysis}

Data were analyzed using IBM SPSS ver. 20 (IBM Corp., Armonk, NY, USA). Descriptive statistics such as frequency, percentage, mean, standard deviation, and inferential statistics such as independent t-test and analysis of variance were used in this study. Kolmogorov-Smirnov test was used to investigate the normal data distribution in each of the measurements. The significance level was set at 0.05 .

\section{RESULTS}

In the overall participant group (263 nurses and 104 physicians), most nurses were female (87.5\%) and most physicians were male ( $88.5 \%, \mathrm{P}=0.001)$. Most nurses were $31-40$ years old (41.4\%), and most physicians were $41-50$ years old (69.2\%) $(\mathrm{P}=0.001)$. The majority of participants among both nurses $(76.6 \%)$ and physicians (99\%) were married ( $\mathrm{P}=0.001)$. The clinical work experience of both nurses (33.7\%) and physicians (36.9\%) was 6-10 years ( $\mathrm{P}=0.67)$. Most nurses had $1-5$ years of work experience in intensive care units (49.6\%), while most physicians had 6-10 years in these units $(35.6 \%)(\mathrm{P}=0.001)$. Most physicians passed an educational course on the nutrition of patients $(84.6 \%)$, while the majority of nurses had not received such a course $(70.0 \%)(\mathrm{P}=0.001)$ (Table 1$)$.

From the perspective of physicians, the barriers to enteral nutrition were dietician support, resources, guideline recommendations, implementation strategies, delivery of enteral nutrition to the patient, and critical care provider attitudes and behavior. From the perspective of nurses, guideline recommendations and implementation strategies, dietician support, resources, delivery of enteral nutrition to the patient, and critical care provider attitudes and behavior were the barriers to enteral nutrition. Although the mean total score of enteral nutrition barriers from the perspective of nurses was less than that of physicians, the difference was not statistically signifi- 
Table 1. Comparison of the demographic characteristics of physicians and nurses in ICUs

\begin{tabular}{|c|c|c|c|c|}
\hline Characteristics & Physician & Nurse & Chi-square test & P-value \\
\hline Sex & & & 191.23 & 0.001 \\
\hline Female & $12(11.5)$ & $230(87.5)$ & & \\
\hline Male & $92(88.5)$ & $33(12.5)$ & & \\
\hline Age (yr) & & & 186.58 & 0.001 \\
\hline $20-30$ & $2(1.9)$ & $103(39.2)$ & & \\
\hline $31-40$ & $5(4.8)$ & $109(41.4)$ & & \\
\hline $41-50$ & $72(69.2)$ & $21(8.0)$ & & \\
\hline$>50$ & $25(24.0)$ & $30(11.4)$ & & \\
\hline Marital status & & & 26.22 & 0.001 \\
\hline Single & $2(1.9)$ & $62(23.6)$ & & \\
\hline Married & $102(98.1)$ & $201(76.4)$ & & \\
\hline Clinical work experience (yr) & & & 1.56 & 0.67 \\
\hline $0-5$ & $27(26.0)$ & $86(32.7)$ & & \\
\hline $6-10$ & 38 (36.5) & $88(33.5)$ & & \\
\hline $11-15$ & $25(24.0)$ & $57(21.7)$ & & \\
\hline$>15$ & $14(13.5)$ & $32(12.2)$ & & \\
\hline Work experience in ICU (yr) & & & 15.56 & 0.001 \\
\hline $1-5$ & $32(30.8)$ & $128(48.7)$ & & \\
\hline $6-10$ & $36(34.6)$ & $87(33.1)$ & & \\
\hline $11-15$ & $21(20.2)$ & $35(13.3)$ & & \\
\hline$>15$ & $15(14.4)$ & $13(5.0)$ & & \\
\hline Educational course of nutrition for patients & & & 89.52 & 0.001 \\
\hline Yes & $88(84.6)$ & $79(30.0)$ & & \\
\hline No & $16(15.4)$ & $184(70.0)$ & & \\
\hline
\end{tabular}

Values are presented as number (\%).

ICU: intensive care unit.

Table 2. Comparison of the perceived mean scores of enteral nutrition barriers in physicians and nurses in ICUs

\begin{tabular}{|c|c|c|c|c|}
\hline Subscale of enteral nutrition barriers & Nurse & Physician & t-test & P-value \\
\hline Guideline recommendations and implementation strategies & $3.91 \pm 0.05$ & $3.77 \pm 0.10$ & 1.27 & 0.205 \\
\hline ICU resources & $3.80 \pm 0.05$ & $3.98 \pm 0.11$ & -1.39 & 0.026 \\
\hline Dietician support & $3.90 \pm 0.05$ & $4.60 \pm 0.04$ & -9.41 & $<0.001$ \\
\hline Delivery of enteral nutrition to the patient & $3.66 \pm 0.05$ & $3.50 \pm 0.05$ & 2.24 & 0.165 \\
\hline Critical care provider attitudes and behavior & $3.52 \pm 0.05$ & $3.33 \pm 0.04$ & 2.71 & 0.007 \\
\hline Total & $3.74 \pm 0.68$ & $3.75 \pm 0.04$ & -0.18 & 0.855 \\
\hline
\end{tabular}

Values are presented as mean \pm standard deviation.

ICU: intensive care unit.

cant $(\mathrm{P}=0.855)$

Among the subscales of enteral nutrition barriers, there was a significant difference among the two groups in the three subscales of resources $(\mathrm{P}=0.026)$, dietician support $(\mathrm{P}<0.001)$, and critical care provider attitudes and behavior $(\mathrm{P}=0.007)$. Regarding resources and dietician support, the highest score was from physicians; regarding critical care provider attitudes and behav- ior, the highest score was from nurses. There was no significant difference between the perspectives of physicians and nurses in guideline recommendations and implementation strategies and delivery of enteral nutrition to the patient (Table 2).

In the case of guideline barriers, the mean score of four questions (including: I am not familiar with our current guidelines for nutrition in the intensive care unit, current scientific 
evidence supporting some nutrition interventions is inadequate to inform practice, the language of the recommendations of the current guidelines for nutrition are not easy to understand, and current feeding protocol is outdated) were significantly higher for nurses than physicians. However, in two questions (including: the current guidelines for nutrition are not readily accessible when I want to refer to them and no feeding protocol in place to guide the initiation and progression of enteral nutrition), the mean score in physicians was significantly higher than in nurses $(\mathrm{P}<0.05)$. In the case of resource barriers, the mean score of the question "no or not enough feeding pumps on the unit" was significantly higher in physicians than in nurses $(\mathrm{P}<0.05)$. In the case of nutritionist barriers, the mean score of all questions (including: waiting for the dietitian to assess the patient, dietitian not routinely present on weekday patient rounds, no or not enough dietitian coverage during evenings, weekends and holidays, and not enough time dedicated to education and training on how to optimally feed patients) was significantly higher in physicians than in nurses $(\mathrm{P}<0.05)$. Regarding barriers to enteral nutrition delivery, the mean score of three questions (including: delay in physicians ordering the initiation of enteral nutrition, waiting for physician/radiology to read X-ray and confirm tube placement, and frequent displacement of feeding tube, requiring reinsertion) was significantly higher in nurses than physicians; for the other three questions (including: delays and difficulties in obtaining small bowel access in patients not tolerating enteral nutrition (i.e. high gastric residual volumes), in resuscitated, hemodynamically stable patients, other aspects of patient care still take priority over, and nutrition therapy not routinely discussed on patient care rounds), the mean score of physicians was significantly higher than nurses $(\mathrm{P}<0.05)$. Regarding the barriers related to nutritional attitude, the mean score of three questions (including: non-intensive care unit physicians (i.e., surgeons, gastroenterologists) requesting patients not be fed enterally, nurses failing to progress feeds as per the feeding protocol, and general belief among intensive care unit team that provision of adequate nutrition does not impact on patient outcome) was significantly higher in nurses than physicians, while for the other three questions (including: feeds being held due to diarrhea, fear of adverse events due to aggressively feeding patients, and feeding being held too far in advance of procedures or operating room visits), the mean score of physicians was significantly higher than physicians $(\mathrm{P}<0.05)$ (Table 3$)$.

These results showed that there was a significant relation- ship between the mean score perceived by physicians and nurses and the educational course. The mean score perceived by physicians and nurses who passed the educational course was significantly higher $(\mathrm{P}<0.05)$.

\section{DISCUSSION}

The results of this study showed that the perceived mean total score of enteral nutrition barriers from the perspective of nurses was lower than that of physicians, but this difference was not statistically significant. These results indicated that the perception and scoring of enteral nutrition barriers of both physicians and nurses were in agreement, which was consistent with the results of Chapple et al [15].

Based on the current results, physicians were considerably more aware of the importance of resources in the intensive care units such as no or not enough feeding pumps on the unit. Therefore, the restriction of resources can be a big barrier for critical care providers to follow guideline recommendations. Consistent with this result, Shayesteh et al. [17] reported that one of the barriers to enteral nutrition was sufficient resources and facilities. For example, in Iran, providing ICU resources imposes high expenses on patients. Lack of budget and insufficient facilities will affect the quality of treatment in intensive care units.

Physicians assigned a higher score to all questions related to nutritionist barriers to dietician support, meaning that from the perspective of physicians, a lack of dietician support can be a big barrier to proper nutrition for intensive care patients. Most physicians indicated that a lack of dietician support can be an important barrier for the proper nutrition of patients hospitalized in intensive care units, and this is consistent with the results of Shayesteh et al. [17]. In addition, a study at Hummer Smith hospital in London showed that dietician support in the intensive care units is so critical that nutrition consultation and diet therapy strategies in these patients lead to a decrease in malnutrition and improvement of weight gain [19]. The American Society for Parenteral Enteral Nutrition stated that dietician support is vital for recovery, an increase of safety, and a decrease of treatment expenses. Hospital managers should take into account nutrition consultation for proper enteral nutrition [20]. Chapple et al. [15] also emphasized the role of dieticians along with physicians and nurses in proper enteral nutrition for patients hospitalized in intensive care units.

Based on the current results, the mean score of critical care provider attitudes and behaviors (such as a request from 
Table 3. Comparison of questions of enteral nutrition barriers in physicians and nurses in ICUs

\begin{tabular}{|c|c|c|c|c|c|}
\hline Subscale & Question of enteral nutrition barriers & Nurse & Physician & t-test & P-value \\
\hline \multirow[t]{6}{*}{$\begin{array}{l}\text { Guideline recommendations and } \\
\text { implementation strategies }\end{array}$} & $\begin{array}{l}\text { I am not familiar with our current guidelines for nutrition in } \\
\text { the ICU. }\end{array}$ & $4.06 \pm 0.06$ & $3.81 \pm 0.11$ & 1.85 & 0.060 \\
\hline & $\begin{array}{l}\text { Current scientific evidence supporting some nutrition } \\
\text { interventions is inadequate to inform practice. }\end{array}$ & $3.80 \pm 0.68$ & $3.50 \pm 0.12$ & 2.22 & 0.020 \\
\hline & $\begin{array}{l}\text { The language of the recommendations of the current } \\
\text { guidelines for nutrition are not easy to understand. }\end{array}$ & $3.65 \pm 0.07$ & $3.22 \pm 0.11$ & 3.24 & 0.001 \\
\hline & $\begin{array}{l}\text { The current guidelines for nutrition are not readily accessible } \\
\text { when I want to refer to them. }\end{array}$ & $3.95 \pm 0.06$ & $4.31 \pm 0.12$ & -2.77 & 0.006 \\
\hline & $\begin{array}{l}\text { No feeding protocol in place to guide the initiation and } \\
\text { progression of enteral nutrition }\end{array}$ & $4.04 \pm 0.06$ & $4.34 \pm 0.12$ & -2.27 & 0.023 \\
\hline & Current feeding protocol is outdated. & $3.94 \pm 0.06$ & $3.46 \pm 0.13$ & 3.62 & 0.001 \\
\hline \multirow[t]{3}{*}{ ICU resources } & Not enough nursing staff to deliver adequate nutrition & $3.39 \pm 0.08$ & $3.27 \pm 0.15$ & 0.70 & 0.487 \\
\hline & Enteral formula not available on the unit & $4.06 \pm 0.06$ & $4.27 \pm 0.12$ & -1.57 & 0.117 \\
\hline & No or not enough feeding pumps on the unit & $3.94 \pm 0.07$ & $4.40 \pm 0.12$ & -3.09 & 0.002 \\
\hline \multirow[t]{4}{*}{ Dietitian support } & Waiting for the dietitian to assess the patient & $3.98 \pm 0.06$ & $4.43 \pm 0.61$ & -3.83 & 0.002 \\
\hline & Dietitian not routinely present on weekday patient rounds & $3.95 \pm 0.06$ & $4.60 \pm 0.05$ & -5.87 & 0.001 \\
\hline & $\begin{array}{l}\text { No or not enough dietitian coverage during evenings, } \\
\text { weekends and holidays }\end{array}$ & $3.85 \pm 0.07$ & $4.75 \pm 0.06$ & -7.10 & 0.001 \\
\hline & $\begin{array}{l}\text { Not enough time dedicated to education and training on } \\
\text { how to optimally feed patients }\end{array}$ & $3.80 \pm 0.07$ & $4.61 \pm 0.07$ & -6.41 & 0.001 \\
\hline \multirow{7}{*}{$\begin{array}{l}\text { Delivery of enteral nutrition to the } \\
\text { patient }\end{array}$} & Delay in physicians ordering the initiation of enteral nutrition & $3.77 \pm 0.06$ & $2.57 \pm 0.12$ & 9.10 & 0.001 \\
\hline & $\begin{array}{l}\text { Waiting for physician/radiology to read X-ray and confirm } \\
\text { tube placement }\end{array}$ & $3.41 \pm 0.08$ & $2.63 \pm 0.08$ & 5.67 & 0.001 \\
\hline & Frequent displacement of feeding tube, requiring reinsertion & $3.06 \pm 0.09$ & $1.85 \pm 0.09$ & 7.28 & 0.001 \\
\hline & $\begin{array}{l}\text { Delays in initiating motility agents in patients not tolerating } \\
\text { enteral nutrition (i.e., high gastric residual volumes) }\end{array}$ & $3.71 \pm 0.07$ & $3.76 \pm 0.09$ & -0.49 & 0.622 \\
\hline & $\begin{array}{l}\text { Delays and difficulties in obtaining small bowel access in } \\
\text { patients not tolerating enteral nutrition (i.e., high gastric } \\
\text { residual volumes) }\end{array}$ & $3.97 \pm 0.06$ & $4.64 \pm 0.07$ & -6.86 & 0.001 \\
\hline & $\begin{array}{l}\text { In resuscitated, hemodynamically stable patients, other } \\
\text { aspects of patient care still take priority over }\end{array}$ & $3.88 \pm 0.07$ & $4.58 \pm 0.07$ & -6.61 & 0.001 \\
\hline & $\begin{array}{l}\text { Nutrition therapy not routinely discussed on patient care } \\
\text { rounds }\end{array}$ & $3.81 \pm 0.07$ & $4.45 \pm 0.07$ & -5.90 & 0.001 \\
\hline \multirow[t]{6}{*}{$\begin{array}{l}\text { Critical care provider attitudes and } \\
\text { behavior }\end{array}$} & $\begin{array}{l}\text { Non-ICU physicians (i.e., surgeons, gastroenterologists) } \\
\text { requesting patients not be fed entirely }\end{array}$ & $3.39 \pm 0.07$ & $2.15 \pm 0.14$ & 7.55 & 0.001 \\
\hline & Nurses failing to progress feeds as per the feeding protocol & $3.25 \pm 0.08$ & $2.55 \pm 0.13$ & 4.57 & 0.001 \\
\hline & Feeds being held due to diarrhea & $3.69 \pm 0.06$ & $4.31 \pm 0.05$ & -6.81 & 0.001 \\
\hline & Fear of adverse events due to aggressively feeding patients & $3.58 \pm 0.07$ & $4.22 \pm 0.06$ & -6.64 & 0.001 \\
\hline & $\begin{array}{l}\text { Feeding being held too far in advance of procedures or } \\
\text { operating room visits }\end{array}$ & $3.96 \pm 0.07$ & $4.71 \pm 0.06$ & -7.57 & 0.001 \\
\hline & $\begin{array}{l}\text { General belief among ICU team that provision of adequate } \\
\text { nutrition does not impact on patient outcome }\end{array}$ & $3.25 \pm 0.08$ & $2.04 \pm 0.09$ & 9.90 & 0.001 \\
\hline
\end{tabular}

Values are presented as mean \pm standard deviation.

ICU: intensive care unit.

non-intensive care physicians for no need for enteral nutrition, the inability of nurses to promote enteral nutrition, according to the nutritional protocol, and the general belief of care providers that providing proper nutrition does not affect the patient's recovery) was significantly higher for nurses than that of physicians. This result indicates that nurses had a better understanding that incomplete awareness or negative attitude of critical care providers towards guideline recommendations might lead not to following the prescribed instructions and the patient would suffer from the side effects. The understanding of this fact may be from nurses' experience of inappropriate actions of nurses and physicians regarding nutrition and 
lack of nutrition guidelines. Chan et al. [21] investigated the performance of 1,203 nurses for tube placement verification, management of high gastric residual volume, and response to nutritional side effects. The results showed that nurses did not have sufficient knowledge and efficiency in tube feeding [21]. Several studies showed that one of the important barriers to enteral nutrition was the poor performance of care providers and the lack of a system to evaluate their performance [22,23]. However, Mehrnosh et al. [24] reported that the nurses' performance for tube feeding was at an average level and considered the accreditation time of hospitals as the reason for the relative improvement in the performance.

Although nurses and physicians differed on some questions in this study, they did not differ significantly overall. There was no significant difference among the perspectives of physicians and nurses in guideline recommendations and implementation strategies and delivery of enteral nutrition to the patient so that the two groups agreed that features of guideline recommendations and methods chosen for their implementation could interfere with their applicability. For example, format, writing, availability, and being up-to-date are issues that may challenge critical care providers. Several studies have confirmed these results. The two groups also agreed that delivery of enteral nutrition to patients could be a barrier to enteral nutrition, meaning that the patient's status plays a role in successfully applying nutrition guidelines and implementing nutrition guidelines for chronic patients may be more difficult. For example, resuscitation or stabilization of hemodynamic status is more preferred than nutrition, and sometimes there will be a gap between the clinic and their knowledge. In this regard, a study by Cahill et al. [11] in North America stated that the most important nutrition barriers from the perspective of nurses were the gap between evidence-based clinical guidelines and what is done in practice.

The mean score of the perceived enteral nutrition barrier was significantly higher in physicians and nurses who passed educational courses, indicating that training had a vital role in increasing the perception of barriers to enteral nutrition. Consistent with these results, Darawad et al. [25] reported that education, the Internet, and nursing faculty were the main sources of enteral nutrition knowledge for nurses, and the authors concluded that awareness of responsibility, backup documentation system, and recording current enteral nutrition could be effective to improve the perception of enteral nutrition barriers and its function. Several studies confirmed this finding $[13,22,25]$.
This study had some limitations. First, the study included a low number of physicians. Second, the participants' data were evaluated by a self-reported questionnaire. Therefore, a larger sample should be used in future studies to improve potential generalizability.

Since perceived enteral nutrition barriers in intensive care units have been reported at an average level for care providers, and while the providers overall showed agreement in the perception and scoring of enteral nutrition barriers, there was a difference in perception between physicians and nurses on some barriers. This suggests that clarifying the reasons for these differences may help establish strategies such as in-service training to increase the knowledge and skills of physicians and nurses and reduce these differences. The cooperation of the treatment team including physicians and nurses is critical in playing an important role to remove these barriers and provide effective enteral nutrition. Deficiency in clinical guideline recommendations, resources, dietician support, delivery of enteral nutrition to the patient, critical care provider attitudes and behavior are considered as enteral nutrition barriers that negatively affect the quality of. Therefore, critical care providers' behavior and attitude towards proper enteral nutrition may be encouraged by providing sufficient resources, proper clinical guidelines, intra-professional cooperation, and continual training. This investigation should also be explored in other populations. Identifying and overcoming the barriers to enteral nutrition in patients in intensive care units will help prevent complications in these patients and improve patient care.

\section{CONFLICT OF INTEREST}

No potential conflict of interest relevant to this article was reported.

\section{ACKNOWLEDGMENTS}

The present article is derived from the M.S thesis by Kerman University of Medical Sciences for critical care nursing. We thank all physicians and nurses in intensive care units.

\section{ORCID}

Masoumeh Mirhosiny https://orcid.org/0000-0003-3157-3808 Mansour Arab ～https://orcid.org/0000-0002-9495-1346 Parvin Mangolian Shahrbabaki https://orcid.org/0000-0003-0539-5390 


\section{AUTHOR CONTRIBUTIONS}

Conceptualization: MM, PMS. Data curation: MM, PMS. Formal analysis: PMS. Methodology: PMS. Project administration: MA. Visualization: MA . Writing-original draft: PMS. Writingreview \& editing: MA, PMS.

\section{REFERENCES}

1. McClave SA, Taylor BE, Martindale RG, Warren MM, Johnson DR, Braunschweig C, et al. Guidelines for the provision and assessment of nutrition support therapy in the adult critically ill patient: Society of Critical Care Medicine (SCCM) and American Society for Parenteral and Enteral Nutrition (A.S.P.E.N.). JPEN J Parenter Enteral Nutr 2016;40:159-211.

2. Lewis SR, Schofield-Robinson OJ, Alderson P, Smith AF. Enteral versus parenteral nutrition and enteral versus a combination of enteral and parenteral nutrition for adults in the intensive care unit. Cochrane Database Syst Rev 2018;6:CD012276.

3. Tappenden KA, Quatrara B, Parkhurst ML, Malone AM, Fanjiang G, Ziegler TR. Critical role of nutrition in improving quality of care: an interdisciplinary call to action to address adult hospital malnutrition. JPEN J Parenter Enteral Nutr 2013;37:48297.

4. Wei X, Day AG, Ouellette-Kuntz H, Heyland DK. The association between nutritional adequacy and long-term outcomes in critically ill patients requiring prolonged mechanical ventilation: a multicenter cohort study. Crit Care Med 2015;43:156979.

5. O'Leary-Kelley C, Bawel-Brinkley K. Nutrition support protocols: enhancing delivery of enteral nutrition. Crit Care Nurse 2017;37:e15-23

6. Ridley E, Davies A, Cooper DJ. Full-feeding with enteral nutrition is not always "full-feeding" in research and clinical practice. JPEN J Parenter Enteral Nutr 2015;39:383.

7. Cho JH. Lack of evidence for a nutritional support team in a trauma intensive care unit? Acute Crit Care 2020;35:205-6.

8. Elke G, van Zanten AR, Lemieux M, McCall M, Jeejeebhoy KN, Kott M, et al. Enteral versus parenteral nutrition in critically ill patients: an updated systematic review and meta-analysis of randomized controlled trials. Crit Care 2016;20:117.

9. Mula C. Nurses' competency and challenges in enteral feeding in the intensive care unit (ICU) and high dependency units (HDU) of a referral hospital, Malawi. Malawi Med J 2014;26:559.

10. Cochrane LJ, Olson CA, Murray S, Dupuis M, Tooman T, Hayes S.
Gaps between knowing and doing: understanding and assessing the barriers to optimal health care. J Contin Educ Health Prof 2007;27:94-102.

11. Cahill NE, Murch L, Cook D, Heyland DK, Canadian Critical Care Trials Group. Barriers to feeding critically ill patients: a multicenter survey of critical care nurses. J Crit Care 2012;27: 727-34.

12. Darawad MW, Alfasfos N, Zaki I, Alnajar M, Hammad S, Samarkandi OA. ICU nurses' perceived barriers to effective enteral nutrition practices: a multicenter survey study. Open Nurs J 2018;12:67-75.

13. Cahill NE, Dhaliwal R, Day AG, Jiang X, Heyland DK. Nutrition therapy in the critical care setting: what is "best achievable" practice? An international multicenter observational study. Crit Care Med 2010;38:395-401.

14. Kim H, Stotts NA, Froelicher ES, Engler MM, Porter C. Why patients in critical care do not receive adequate enteral nutrition? A review of the literature. J Crit Care 2012;27:702-13.

15. Chapple LA, Chapman M, Shalit N, Udy A, Deane A, Williams L. Barriers to nutrition intervention for patients with a traumatic brain injury: views and attitudes of medical and nursing practitioners in the acute care setting. JPEN J Parenter Enteral Nutr 2018;42:318-26

16. Cahill NE, Suurdt J, Ouellette-Kuntz H, Heyland DK. Understanding adherence to guidelines in the intensive care unit: development of a comprehensive framework. JPEN J Parenter Enteral Nutr 2010;34:616-24.

17. Shayesteh F, Poudineh S, Pouryazdanpanah-Kermani M, Sadat Ayoudi S, Norouzy A. Assessment of nutritional intake in intensive care unit patients of Ghaem hospital. Med J Mashhad Univ Med Sci 2015;58:217-24

18. Cahill NE, Jiang X, Heyland DK. Revised questionnaire to assess barriers to adequate nutrition in the critically ill. JPEN J Parenter Enteral Nutr 2016;40:511-8.

19. O'Flynn J, Peake H, Hickson M, Foster D, Frost G. The prevalence of malnutrition in hospitals can be reduced: results from three consecutive cross-sectional studies. Clin Nutr 2005;24:1078-88

20. ASPEN Board of Directors and the Clinical Guidelines Task Force. Guidelines for the use of parenteral and enteral nutrition in adult and pediatric patients. JPEN J Parenter Enteral Nutr 2002;26(1 Suppl):1SA-138SA.

21. Chan EY, Ng IH, Tan SL, Jabin K, Lee LN, Ang CC. Nasogastric feeding practices: a survey using clinical scenarios. Int J Nurs Stud 2012;49:310-9.

22. Ashouri E, Fatehi N. A comparison of performing tube feeding 
with the standard procedures at selected educational and treatment centers of Isfahan University of Medical Sciences, Iran. Iran J Nurs Midwifery Res 2012;17(2 Suppl 1):S80-4.

23. Zahra ZS, Ahmadli R, Maleki M, Jambarsang S, Dabirian A. Knowledge assessment and comparing the performance of intensive care unit nurses in regard to tube feeding with existing standards in educational and treatment centers of Qom University of Medical Sciences, Iran. Qom Univ Med Sci J
2016;10:45-54.

24. Mehrnosh N, Alipour H, Karimollahi M. Performance of critical care nurses in nasogastric tube nutrition in Ardabil Hospitals. J Health Care 2018;20:186-95.

25. Darawad MW, Hammad S, Al-Hussami M, Haourani E, Aboshaiqah AE, Hamdan-Mansour AM. Investigating critical care nurses' perception regarding enteral nutrition. Nurse Educ Today 2015;35:414-9. 